Article

\title{
A Novel Similarity Measure for Interval-Valued Intuitionistic Fuzzy Sets and Its Applications
}

\author{
Minxia Luo *(1) and Jingjing Liang \\ Department of Information and Computing Science, China Jiliang University, Hangzhou 310018, China; \\ s1608070103@cjlu.edu.cn \\ * Correspondence: mxluo@cjlu.edu.cn; Tel.: +86-571-8687-5634
}

Received: 29 August 2018; Accepted: 25 September 2018; Published: 27 September 2018

\begin{abstract}
In this paper, a novel similarity measure for interval-valued intuitionistic fuzzy sets is introduced, which is based on the transformed interval-valued intuitionistic triangle fuzzy numbers. Its superiority is shown by comparing the proposed similarity measure with some existing similarity measures by some numerical examples. Furthermore, the proposed similarity measure is applied to deal with pattern recognition and medical diagnosis problems.
\end{abstract}

Keywords: interval-valued intuitionistic fuzzy set; similarity measure; pattern recognition

\section{Introduction}

As a generalization concept of fuzzy set (FS) introduced by Zadeh [1], the definition of intuitionistic fuzzy set (IFS) was initiated by Atanassov [2] for dealing with vague and uncertain information, which elaborately describe uncertain information by membership degree, non-membership degree and hesitancy degree. In [3], Gau and Buehrer presented the definition of vague set. In [4], Bustince and Burillo have showed that the notion of IFSs and vague sets coincide with each other. In order to deal with indeterminate and inconsistent information, Smarandache [5] proposed a neutrosophic set (NS). In the NS, indeterminacy-membership $I_{A}(x)$ is independent, thus making the NS more flexible and the most suitable for solving some decision-making problems related to the use of incomplete and imprecise information, uncertainties, predictions and so on. Zhang [6,7] studied algebraic and lattice structure for neutrosophic sets.

The conception of similarity measure for IFSs is one of the most important subjects for degree of similarity between objects in IFS theory. Chen [8] proposed the similarity measure based on a vague set for the first time. Hong [9] introduced a new similarity measure based on vague set and overcame some drawbacks of Chen's similarity measure. Szmidt and Kacprzyk [10] extend Hamming distance and Euclidean distance to construct intuitionistic fuzzy similarity measure. However, Wang and Xin [11] implied that Szmidt and Kacprzyk's distance measure [10] were ineffective in some situations. Grzegorzewski [12] extended some novel similarity measures for IFSs based on Hausdorff distance. Chen [13] pointed out some defects of Grzegorzewski's similarity measure and show some counter examples. On the other hand, some studies defined new similarity measures for IFSs, rather than extending the well-known distance measures. Li and Cheng [14] presented a new similarity measure between IFSs and applied it to pattern recognition. Mitchell [15] indicated that similarity measure of $\mathrm{Li}$ and Cheng [14] had some counter-intuitive cases and modified that similarity measure based on a statistical perspective. Furthermore, Liang and Shi [16] presented some counter instances to indicate that the similarity measure of $\mathrm{Li}$ and Cheng [14] was not suitable for some situations, and proposed several new similarity measures for IFS. Ye [17] conducted a similarity comparative study of existing similarity measures for IFSs and proposed a cosine similarity measure and weighted cosine similarity measure. $\mathrm{Xu}$ [18] acquainted a sequence of similarity measures for IFSs and applied 
to solve multiple attribute decision-making problems. Boran et al. [19] proposed a new general type of similarity measures for IFSs with two parameters, expressing $L_{p}$-norm and give its relation with existing similarity measures. Zhang and Yu [20] presented a new distance measure based on interval comparison, where the IFSs were respectively transformed into the symmetric triangular fuzzy numbers. Comparison with the widely used methods indicated that the proposed method contained more information, with much less loss of information. Luo and Zhao [21] proposed a new distance measure for IFSs, which is based on a matrix norm and a strictly increasing (or decreasing) binary function, and applied it to solve pattern recognition problems.

As the development of IFSs, Atanassov introduced interval-valued intuitionistic fuzzy set (IVIFS) [22], which the membership degree, non-membership degree and hesitancy degree are represented by subinterval of $[0,1]$. It therefore can represent the dynamic character of features accurately. Due to the advantages of IVIFSs in practical application, various similarity measures based on IVIFSs were studied extensively by many researchers from different angles and applied to many areas such as medical diagnosis, pattern recognition problem and so on. Liu [23] proposed a set of axiomatic definitions for entropy measures between IVIFSs, which extends Szmidt and Kacprzyk's axioms formulated for entropy between IFSs. Xu [24] generalized some formulas of similarity measures of IFSs to IVIFSs. Wei [25] proposed an new similarity measure for IVIFSs, and also applied to solve problems on pattern recognitions, multi-criteria fuzzy decision-making and medical diagnosis. Singh [26] introduced a new cosine similarity measure for IVIFSs and applied to pattern recognition. Khalaf [27] advanced a new approach for medical diagnosis by IVIFSs, which is generalized by the application of IFS theory. Dhivya [28] presented a new similarity measure for IVIFSs based on the mid points of transformed triangular fuzzy numbers.

However, there are some drawbacks in some existing similarity measures for IVIFSs, most of which get counterintuitive results in some situations and they cannot get correct classification results for dealing with the pattern recognition problems and medical diagnosis problems. For example, letting $A=<[0.20,0.30],[0.40,0.60]>, B_{1}=<[0.30,0.40],[0.40,0.60]>$ and $B_{2}=<$ $[0.30,0.40],[0.30,0.50]>$ be IVIFSs, we can compute the similarity measures between $A$ and $B_{i}$ $(i=1,2)$ by Formulas (1), (2) and (4) (see Section 3). Obviously, we have the result $B_{1} \neq B_{2}$ because the membership degree of $B_{1}$ is identical to that of $B_{2}$, and the non-membership degree of $B_{1}$ is not identical to that of $B_{2}$. Therefore, we should obtain $S_{i}\left(A, B_{1}\right) \neq S_{i}\left(A, B_{2}\right)(i=1,2)$. However, we can obtain that $S_{1}\left(A, B_{1}\right)=S_{1}\left(A, B_{2}\right)=S_{2}\left(A, B_{1}\right)=S_{2}\left(A, B_{2}\right)=0.9$ by the Formulas (1) and (2) (for $\left.p=1\right)$, which is not reasonable. Meanwhile, we can get $S_{D}\left(A, B_{1}\right)=1$ by Formula (4), which does not satisfy the second axiom of the definition for similarity measure. Therefore, we need to develop a new similarity measure to overcome these drawbacks.

The rest of the paper is organized as follows: Section 2 reviews some necessary definitions related to IVIFS. In Section 3, some existing similarity measures are reviewed. In Section 4, a novel similarity measure is introduced. The geometric interpretation of the new similarity measure and the explanation of parameters are briefly given in Section 5. Applications in pattern recognition and medical diagnosis are presented in Section 6. The conclusions for this paper are given in the last section.

\section{Preliminary}

In this section, we review the basic concepts related to IVIFSs that will be used in this paper.

Definition 1 ([1]). A fuzzy set $A$ in the unverse of discourse $X=\left\{x_{1}, x_{2}, \ldots, x_{n}\right\}$ is defined as follows:

$$
A=\left\{<x, \mu_{A}(x)>\mid x \in X\right\},
$$

where $\mu_{A}(x): X \rightarrow[0,1]$ is the membership degree. 
Definition 2 ([2]). An intuitionistic fuzzy set $A$ in a universe of discourse $X=\left\{x_{1}, x_{2}, \ldots, x_{n}\right\}$ is defined as follows:

$$
A=\left\{<x, \mu_{A}(x), v_{A}(x)>\mid x \in X\right\},
$$

where $\mu_{A}(x): X \rightarrow[0,1]$ and $v_{A}(x): X \rightarrow[0,1]$ are membership and non-membership degree, respectively, such that: $0 \leq \mu_{A}(x)+v_{A}(x) \leq 1$.

The third parameter of intuitionistic fuzzy set $A$ is: $\pi_{A}(x)=1-\mu_{A}(x)-v_{A}(x)$, which is known as the intuitionistic fuzzy index or the hesitation degree of whether $x$ belongs to A or not. It is obviously seen that $0 \leq \pi_{A}(x) \leq 1$. If $\pi_{A}(x)$ is small; then, knowledge about $x$ is more certain; if $\pi_{A}(x)$ is great, then knowledge about $x$ is more uncertain.

Definition 3 ([22]). An interval-valued intuitionistic fuzzy set $A$ in a universe of discourse $X=$ $\left\{x_{1}, x_{2}, \ldots, x_{n}\right\}$ is defined as follows:

$$
A=\left\{<x, \mu_{A}(x), v_{A}(x)>\mid x \in X\right\}=\left\{<x,\left[\mu_{A}^{-}(x), \mu_{A}^{+}(x)\right],\left[v_{A}^{-}(x), v_{A}^{+}(x)\right]>\mid x \in X\right\},
$$

where $\mu_{A}(x) \subseteq[0,1], v_{A}(x) \subseteq[0,1]$, which satisfies $0 \leq \mu_{A}^{+}(x)+v_{A}^{+}(x) \leq 1$.

The intervals $\mu_{A}(x)$ and $v_{A}(x)$ denote the membership degree and non-membership degree, respectively. Furthermore, for each $x \in X$, we can compute the hesitance degree $\pi_{A}(x)=\left[\pi_{A}^{-}\left(x_{i}\right), \pi_{A}^{+}\left(x_{i}\right)\right]=[1-$ $\left.\mu_{A}^{+}(x)-v_{A}^{+}(x), 1-\mu_{A}^{-}(x)-v_{A}^{-}(x)\right]$.

Definition 4 ([29]). For every two IVIFSs $A$ and B in the universe of discourse X, we have the following relations:

(1): $A \subseteq B$ iff $(\forall x \in X) \mu_{A}^{-}(x) \leq \mu_{B}^{-}(x)$ and $\mu_{A}^{+}(x) \leq \mu_{B}^{+}(x)$ and $v_{A}^{-}(x) \geq v_{B}^{-}(x)$ and $v_{A}^{+}(x) \geq v_{B}^{+}(x)$.

(2): $\quad A \cup B=\left\langle x,\left[\max \left(\mu_{A}^{-}(x), \mu_{B}^{-}(x)\right), \max \left(\mu_{A}^{+}(x), \mu_{B}^{+}(x)\right)\right],\left[\min \left(v_{A}^{-}(x), v_{B}^{-}(x)\right), \min \left(v_{A}^{+}(x), v_{B}^{+}(x)\right)\right]\right\rangle$.

(3): $A \cap B=\left\langle x,\left[\min \left(\mu_{A}^{-}(x), \mu_{B}^{-}(x)\right), \min \left(\mu_{A}^{+}(x), \mu_{B}^{+}(x)\right)\right],\left[\max \left(v_{A}^{-}(x), v_{B}^{-}(x)\right), \max \left(v_{A}^{+}(x), v_{B}^{+}(x)\right)\right]\right\rangle$.

(4): $\quad A=B$ iff $(\forall x \in X) \mu_{A}^{-}(x)=\mu_{B}^{-}(x)$ and $\mu_{A}^{+}(x)=\mu_{B}^{+}(x)$ and $v_{A}^{-}(x)=v_{B}^{-}(x)$ and $v_{A}^{+}(x)=v_{B}^{+}(x)$.

(5): $\quad A^{c}=\left\langle x,\left[v_{A}^{-}(x), v_{A}^{+}(x)\right],\left[\mu_{A}^{-}(x), \mu_{A}^{+}(x)\right]\right\rangle$

Definition 5 ([18]). Let $A$ and $B$ be interval-valued intuitionistic fuzzy sets in the unverse of discourse $X=\left\{x_{1}, x_{2}, \ldots, x_{n}\right\}$, a mapping $S: \operatorname{IVIFS}(X) \times \operatorname{IVIFS}(X) \rightarrow[0,1], S(A, B)$ is called to be a similarity measure between $A$ and $B$, if $S(A, B)$ satisfies the following properties:

(S1): $\quad 0 \leq S(A, B) \leq 1$,

(S2): $S(A, B)=1$ if and only if $A=B$,

(S3): $\quad S(A, B)=S(B, A)$,

(S4): If $A \subseteq B \subseteq C$, then $S(A, C) \leq S(A, B)$, and $S(A, C) \leq S(B, C)$.

\section{Some Existing Similarity Measures}

In this section, we review some existing similarity measures.

Let $A=\left\{<x_{i},\left[\mu_{A}^{-}\left(x_{i}\right), \mu_{A}^{+}\left(x_{i}\right)\right],\left[v_{A}^{-}\left(x_{i}\right), v_{A}^{+}\left(x_{i}\right)\right]>\mid x_{i} \in X\right\}, B=\left\{<x_{i},\left[\mu_{B}^{-}\left(x_{i}\right), \mu_{B}^{+}\left(x_{i}\right)\right],\left[v_{B}^{-}\left(x_{i}\right)\right.\right.$, $\left.\left.v_{B}^{+}\left(x_{i}\right)\right]>\mid x_{i} \in X\right\}$ be IVIFSs defined on a universe of discourse $X=\left\{x_{1}, x_{2}, \ldots, x_{n}\right\}$. The following Formulas (1)-(4) are similarity measures based on IVIFSs:

$\mathrm{Xu}$ 's similarity measure([24]):

$$
\begin{aligned}
& S_{1}(A, B)=1-\sqrt[p]{\frac{1}{4 n} \sum_{i=1}^{n}\left(\left|\mu_{A}^{-}\left(x_{i}\right)-\mu_{B}^{-}\left(x_{i}\right)\right|^{p}+\left|\mu_{A}^{+}\left(x_{i}\right)-\mu_{B}^{+}\left(x_{i}\right)\right|^{p}+\left|v_{A}^{-}\left(x_{i}\right)-v_{B}^{-}\left(x_{i}\right)\right|^{p}+\left|v_{A}^{+}\left(x_{i}\right)-v_{B}^{+}\left(x_{i}\right)\right|^{p}\right)}, \\
& S_{2}(A, B)=1-\sqrt[p]{\frac{1}{n} \sum_{i=1}^{n} \max \left(\left|\mu_{A}^{-}\left(x_{i}\right)-\mu_{B}^{-}\left(x_{i}\right)\right|^{p},\left|\mu_{A}^{+}\left(x_{i}\right)-\mu_{B}^{+}\left(x_{i}\right)\right|^{p},\left|v_{A}^{-}\left(x_{i}\right)-v_{B}^{-}\left(x_{i}\right)\right|^{p},\left|v_{A}^{+}\left(x_{i}\right)-v_{B}^{+}\left(x_{i}\right)\right|^{p}\right)} .
\end{aligned}
$$


Wei's similarity measure ([25]):

$$
S_{W}(A, B)=\frac{1}{n} \sum_{i=1}^{n} \frac{2-\min \left(\mu_{i}^{-}, v_{i}^{-}\right)-\min \left(\mu_{i}^{+}, v_{i}^{+}\right)}{2+\max \left(\mu_{i}^{-}, v_{i}^{-}\right)+\max \left(\mu_{i}^{+}, v_{i}^{+}\right)}
$$

where

$$
\begin{aligned}
& \mu_{i}^{-}=\left|\mu_{A}^{-}\left(x_{i}\right)-\mu_{B}^{-}\left(x_{i}\right)\right|, \mu_{i}^{+}=\left|\mu_{A}^{+}\left(x_{i}\right)-\mu_{B}^{+}\left(x_{i}\right)\right|, \\
& v_{i}^{-}=\left|v_{A}^{-}\left(x_{i}\right)-v_{B}^{-}\left(x_{i}\right)\right|, v_{i}^{+}=\left|v_{A}^{+}\left(x_{i}\right)-v_{B}^{+}\left(x_{i}\right)\right| .
\end{aligned}
$$

Dhivya's similarity measure ([28]):

$$
\begin{aligned}
S_{D}(A, B)= & 1-\frac{1}{n} \sum_{i=1}^{n}\left(\frac{1}{2}\left(\left|\psi_{A}^{-}\left(x_{i}\right)-\psi_{B}^{-}\left(x_{i}\right)\right|+\left|\psi_{A}^{+}\left(x_{i}\right)-\psi_{B}^{+}\left(x_{i}\right)\right|\right) \cdot\left(1-\frac{\sigma_{A}\left(x_{i}\right)+\sigma_{B}\left(x_{i}\right)}{2}\right)+\right. \\
& \left.\left|\sigma_{A}\left(x_{i}\right)-\sigma_{B}\left(x_{i}\right)\right| \cdot\left(\frac{\sigma_{A}\left(x_{i}\right)+\sigma_{B}\left(x_{i}\right)}{2}\right)\right)
\end{aligned}
$$

where

$$
\begin{aligned}
& \psi_{A}^{-}=\frac{\mu_{A}^{-}\left(x_{i}\right)+1-v_{A}^{-}\left(x_{i}\right)}{2}, \psi_{A}^{+}=\frac{\mu_{A}^{+}\left(x_{i}\right)+1-v_{A}^{+}\left(x_{i}\right)}{2}, \\
& \psi_{B}^{-}=\frac{\mu_{B}^{-}\left(x_{i}\right)+1-v_{B}^{-}\left(x_{i}\right)}{2}, \psi_{B}^{+}=\frac{\mu_{B}^{+} v+1-v_{B}^{+}\left(x_{i}\right)}{2}, \\
& \sigma_{A}\left(x_{i}\right)=1-\frac{1}{2}\left(\mu_{A}^{-}\left(x_{i}\right)+\mu_{A}^{+}\left(x_{i}\right)+v_{A}^{-}\left(x_{i}\right)+v_{A}^{+}\left(x_{i}\right)\right), \\
& \sigma_{B}\left(x_{i}\right)=1-\frac{1}{2}\left(\mu_{B}^{-}\left(x_{i}\right)+\mu_{B}^{+}\left(x_{i}\right)+v_{B}^{-}\left(x_{i}\right)+v_{B}^{+}\left(x_{i}\right)\right) .
\end{aligned}
$$

\section{A New Similarity Measure between Interval-Valued Intuitionistic Fuzzy Sets}

Definition 6. Let $A, B$ be IVIFSs defined in universe of discourse $X=\left\{x_{1}, x_{2}, \ldots, x_{n}\right\}$, and $A=\{<$ $\left.x_{i},\left[\mu_{A}^{-}\left(x_{i}\right), \mu_{A}^{+}\left(x_{i}\right)\right],\left[v_{A}^{-}\left(x_{i}\right), v_{A}^{+}\left(x_{i}\right)\right]>\mid x_{i} \in X\right\}, B=\left\{<x_{i},\left[\mu_{B}^{-}\left(x_{i}\right), \mu_{B}^{+}\left(x_{i}\right)\right],\left[v_{B}^{-}\left(x_{i}\right), v_{B}^{+}\left(x_{i}\right)\right]>\mid x_{i} \in X\right\}$. We call

$$
S^{p}(A, B)=1-\left\{\begin{array}{l}
\frac{1}{2 n} \sum_{i=1}^{n}\left|\frac{t_{1}\left[\left(\mu_{A}^{-}\left(x_{i}\right)-\mu_{B}^{-}\left(x_{i}\right)\right)+\left(\mu_{A}^{+}\left(x_{i}\right)-\mu_{B}^{+}\left(x_{i}\right)\right)\right]-\left[\left(v_{A}^{-}\left(x_{i}\right)-v_{B}^{-}\left(x_{i}\right)\right)+\left(v_{A}^{+}\left(x_{i}\right)-v_{B}^{+}\left(x_{i}\right)\right)\right]}{2\left(t_{1}+1\right)}\right|^{p} \\
+\left|\frac{t_{2}\left[\left(v_{A}^{-}\left(x_{i}\right)-v_{B}^{-}\left(x_{i}\right)\right)+\left(v_{A}^{+}\left(x_{i}\right)-v_{B}^{+}\left(x_{i}\right)\right)\right]-\left[\left(\mu_{A}^{-}\left(x_{i}\right)-\mu_{B}^{-}\left(x_{i}\right)\right)+\left(\mu_{A}^{+}\left(x_{i}\right)-\mu_{B}^{+}\left(x_{i}\right)\right)\right]}{2\left(t_{2}+1\right)}\right|^{p}
\end{array}\right\}
$$

a similarity measure between $A$ and $B . t_{1}, t_{2}, p \in[1,+\infty)$. Here, three parameters: $p$ is the $L_{p}$-norm and $t_{1}, t_{2}$ identifies the level of uncertainty.

Theorem 1. $S^{p}(A, B)$ is a similarity measure between IVIFSs $A$ and $B$.

Proof. Let $A, B, C$ be IVIFSs defined on a universe of discourse $X=\left\{x_{1}, x_{2}, \ldots, x_{n}\right\}$, and $A=\{<$ $\left.x_{i},\left[\mu_{A}^{-}\left(x_{i}\right), \mu_{A}^{+}\left(x_{i}\right)\right],\left[v_{A}^{-}\left(x_{i}\right), v_{A}^{+}\left(x_{i}\right)\right]>\mid x_{i} \in X\right\}, B=\left\{<x_{i},\left[\mu_{B}^{-}\left(x_{i}\right), \mu_{B}^{+}\left(x_{i}\right)\right],\left[v_{B}^{-}\left(x_{i}\right), v_{B}^{+}\left(x_{i}\right)\right]>\mid x_{i} \in X\right\}$, and $C=\left\{<x_{i},\left[\mu_{C}^{-}\left(x_{i}\right), \mu_{C}^{+}\left(x_{i}\right)\right],\left[v_{C}^{-}\left(x_{i}\right), v_{C}^{+}\left(x_{i}\right)\right]>\mid x_{i} \in X\right\}$.

(1) Firstly, we know that, for arbitrary $x_{i} \in X$ :

$$
\begin{aligned}
& t_{1}\left[\left(\mu_{A}^{-}\left(x_{i}\right)-\mu_{B}^{-}\left(x_{i}\right)\right)+\left(\mu_{A}^{+}\left(x_{i}\right)-\mu_{B}^{+}\left(x_{i}\right)\right)\right]-\left[\left(v_{A}^{-}\left(x_{i}\right)-v_{B}^{-}\left(x_{i}\right)\right)+\left(v_{A}^{+}\left(x_{i}\right)-v_{B}^{+}\left(x_{i}\right)\right)\right] \\
= & {\left[t_{1}\left(\mu_{A}^{-}\left(x_{i}\right)-\mu_{B}^{-}\left(x_{i}\right)\right)-\left(v_{A}^{-}\left(x_{i}\right)-v_{B}^{-}\left(x_{i}\right)\right)\right]+\left[t_{1}\left(\mu_{A}^{+}\left(x_{i}\right)-\mu_{B}^{+}\left(x_{i}\right)\right)-\left(v_{A}^{+}\left(x_{i}\right)-v_{B}^{+}\left(x_{i}\right)\right)\right] . }
\end{aligned}
$$


For $\mu_{A}^{-}\left(x_{i}\right), \mu_{B}^{-}\left(x_{i}\right), v_{A}^{-}\left(x_{i}\right), v_{B}^{-}\left(x_{i}\right) \in[0,1]$, then we have $-t_{1} \leq t_{1}\left(\mu_{A}^{-}\left(x_{i}\right)-\mu_{B}^{-}\left(x_{i}\right)\right) \leq t_{1}$, $-1 \leq v_{A}^{-}\left(x_{i}\right)-v_{B}^{-}\left(x_{i}\right) \leq 1$. Thus, we obtain that

$$
-\left(t_{1}+1\right) \leq t_{1}\left(\mu_{A}^{-}\left(x_{i}\right)-\mu_{B}^{-}\left(x_{i}\right)\right)-\left(v_{A}^{-}\left(x_{i}\right)-v_{B}^{-}\left(x_{i}\right)\right) \leq t_{1}+1 .
$$

Similarly,

$$
-\left(t_{1}+1\right) \leq t_{1}\left(\mu_{A}^{+}\left(x_{i}\right)-\mu_{B}^{+}\left(x_{i}\right)\right)-\left(v_{A}^{+}\left(x_{i}\right)-v_{B}^{+}\left(x_{i}\right)\right) \leq t_{1}+1 .
$$

Thus,

$$
0 \leq\left|\frac{t_{1}\left[\left(\mu_{A}^{-}\left(x_{i}\right)-\mu_{B}^{-}\left(x_{i}\right)\right)+\left(\mu_{A}^{+}\left(x_{i}\right)-\mu_{B}^{+}\left(x_{i}\right)\right)\right]-\left[\left(v_{A}^{-}\left(x_{i}\right)-v_{B}^{-}\left(x_{i}\right)\right)+\left(v_{A}^{+}\left(x_{i}\right)-v_{B}^{+}\left(x_{i}\right)\right)\right]}{2\left(t_{1}+1\right)}\right|^{p} \leq 1 .
$$

By the same way, we have

$$
0 \leq\left|\frac{t_{2}\left[\left(v_{A}^{-}\left(x_{i}\right)-v_{B}^{-}\left(x_{i}\right)\right)+\left(v_{A}^{+}\left(x_{i}\right)-v_{B}^{+}\left(x_{i}\right)\right)\right]-\left[\left(\mu_{A}^{-}\left(x_{i}\right)-\mu_{B}^{-}\left(x_{i}\right)\right)+\left(\mu_{A}^{+}\left(x_{i}\right)-\mu_{B}^{+}\left(x_{i}\right)\right)\right]}{2\left(t_{2}+1\right)}\right|^{p} \leq 1 .
$$

Therefore,

$$
0 \leq\left\{\begin{array}{l}
\frac{1}{2 n} \sum_{i=1}^{n}\left|\frac{t_{1}\left[\left(\mu_{A}^{-}\left(x_{i}\right)-\mu_{B}^{-}\left(x_{i}\right)\right)+\left(\mu_{A}^{+}\left(x_{i}\right)-\mu_{B}^{+}\left(x_{i}\right)\right)\right]-\left[\left(v_{A}^{-}\left(x_{i}\right)-v_{B}^{-}\left(x_{i}\right)\right)+\left(v_{A}^{+}\left(x_{i}\right)-v_{B}^{+}\left(x_{i}\right)\right)\right]}{2\left(t_{1}+1\right)}\right|^{p} \\
+\left|\frac{t_{2}\left[\left(v_{A}^{-}\left(x_{i}\right)-v_{B}^{-}\left(x_{i}\right)\right)+\left(v_{A}^{+}\left(x_{i}\right)-v_{B}^{+}\left(x_{i}\right)\right)\right]-\left[\left(\mu_{A}^{-}\left(x_{i}\right)-\mu_{B}^{-}\left(x_{i}\right)\right)+\left(\mu_{A}^{+}\left(x_{i}\right)-\mu_{B}^{+}\left(x_{i}\right)\right)\right]}{2\left(t_{2}+1\right)}\right|^{p}
\end{array}\right\} \leq 1 .
$$

That is, $0 \leq S^{p}(A, B) \leq 1$.

(2) $A=B$, if and only if for arbitrary $x_{i} \in X$, we have $\mu_{A}^{-}\left(x_{i}\right)=\mu_{B}^{-}\left(x_{i}\right), \mu_{A}^{+}\left(x_{i}\right)=\mu_{B}^{+}\left(x_{i}\right)$, $v_{A}^{-}\left(x_{i}\right)=v_{B}^{-}\left(x_{i}\right), v_{A}^{+}\left(x_{i}\right)=v_{B}^{+}\left(x_{i}\right)$. It is obvious that $S^{p}(A, B)=1$.

(3) For $S^{p}(A, B)$, we have

$$
\begin{aligned}
& \left|t_{1}\left[\left(\mu_{A}^{-}\left(x_{i}\right)-\mu_{B}^{-}\left(x_{i}\right)\right)+\left(\mu_{A}^{+}\left(x_{i}\right)-\mu_{B}^{+}\left(x_{i}\right)\right)\right]-\left[\left(v_{A}^{-}\left(x_{i}\right)-v_{B}^{-}\left(x_{i}\right)\right)+\left(v_{A}^{+}\left(x_{i}\right)-v_{B}^{+}\left(x_{i}\right)\right)\right]\right|^{p} \\
= & \left|-t_{1}\left[\left(\mu_{A}^{-}\left(x_{i}\right)-\mu_{B}^{-}\left(x_{i}\right)\right)+\left(\mu_{A}^{+}\left(x_{i}\right)-\mu_{B}^{+}\left(x_{i}\right)\right)\right]+\left[\left(v_{A}^{-}\left(x_{i}\right)-v_{B}^{-}\left(x_{i}\right)\right)+\left(v_{A}^{+}\left(x_{i}\right)-v_{B}^{+} v\right)\right]\right|^{p} \\
= & \left|t_{1}\left[\left(\mu_{B}^{-}\left(x_{i}\right)-\mu_{A}^{-}\left(x_{i}\right)\right)+\left(\mu_{B}^{+}\left(x_{i}\right)-\mu_{A}^{+}\left(x_{i}\right)\right)\right]-\left[\left(v_{B}^{-}\left(x_{i}\right)-v_{A}^{-}\left(x_{i}\right)\right)-\left(v_{B}^{+}\left(x_{i}\right)-v_{A}^{+}\left(x_{i}\right)\right)\right]\right|^{p} .
\end{aligned}
$$

Similarly,

$$
\begin{aligned}
& \left|t_{2}\left[\left(v_{A}^{-}\left(x_{i}\right)-v_{B}^{-}\left(x_{i}\right)\right)+\left(v_{A}^{+}\left(x_{i}\right)-v_{B}^{+}\left(x_{i}\right)\right)\right]-\left[\left(\mu_{A}^{-}\left(x_{i}\right)-\mu_{B}^{-}\left(x_{i}\right)\right)+\left(\mu_{A}^{+}\left(x_{i}\right)-\mu_{B}^{+}\left(x_{i}\right)\right)\right]\right|^{p} \\
= & \left|-t_{2}\left[\left(v_{A}^{-}\left(x_{i}\right)-v_{B}^{-}\left(x_{i}\right)\right)+\left(v_{A}^{+}\left(x_{i}\right)-v_{B}^{+}\left(x_{i}\right)\right)\right]+\left[\left(\mu_{A}^{-}\left(x_{i}\right)-\mu_{B}^{-}\left(x_{i}\right)\right)+\left(\mu_{A}^{+}\left(x_{i}\right)-\mu_{B}^{+}\left(x_{i}\right)\right)\right]\right|^{p} \\
= & \left|t_{2}\left[\left(v_{B}^{-}\left(x_{i}\right)-v_{A}^{-}\left(x_{i}\right)\right)-\left(v_{B}^{+}\left(x_{i}\right)-v_{A}^{+}\left(x_{i}\right)\right)\right]-\left[\left(\mu_{B}^{-}\left(x_{i}\right)-\mu_{A}^{-}\left(x_{i}\right)\right)-\left(\mu_{B}^{+}\left(x_{i}\right)-\mu_{A}^{+}\left(x_{i}\right)\right)\right]\right|^{p} .
\end{aligned}
$$

Thus, $S^{p}(A, B)=S^{p}(B, A)$.

(4) For $A, B, C$ be IVIFSs, the similarity measure $A$ and $B$, and $A$ and $C$ are the following:

$$
\begin{aligned}
& S^{p}(A, B)=1-\left\{\begin{array}{l}
\frac{1}{2 n} \sum_{i=1}^{n}\left|\frac{t_{1}\left[\left(\mu_{A}^{-}\left(x_{i}\right)-\mu_{B}^{-}\left(x_{i}\right)\right)+\left(\mu_{A}^{+}\left(x_{i}\right)-\mu_{B}^{+}\left(x_{i}\right)\right)\right]-\left[\left(v_{A}^{-}\left(x_{i}\right)-v_{B}^{-}\left(x_{i}\right)\right)+\left(v_{A}^{+}\left(x_{i}\right)-v_{B}^{+}\left(x_{i}\right)\right)\right]}{2\left(t_{1}+1\right)}\right|^{p} \\
+\left|\frac{t_{2}\left[\left(v_{A}^{-}\left(x_{i}\right)-v_{B}^{-}\left(x_{i}\right)\right)+\left(v_{A}^{+}\left(x_{i}\right)-v_{B}^{+}\left(x_{i}\right)\right)\right]-\left[\left(\mu_{A}^{-}\left(x_{i}\right)-\mu_{B}^{-}\left(x_{i}\right)\right)+\left(\mu_{A}^{+}\left(x_{i}\right)-\mu_{B}^{+}\left(x_{i}\right)\right)\right]}{2\left(t_{2}+1\right)}\right|^{p}
\end{array}\right\}^{\frac{1}{p}}, \\
& S^{p}(A, C)=1-\left\{\begin{array}{l}
\frac{1}{2 n} \sum_{i=1}^{n}\left|\frac{t_{1}\left[\left(\mu_{A}^{-}\left(x_{i}\right)-\mu_{C}^{-}\left(x_{i}\right)\right)+\left(\mu_{A}^{+}\left(x_{i}\right)-\mu_{C}^{+}\left(x_{i}\right)\right)\right]-\left[\left(v_{A}^{-}\left(x_{i}\right)-v_{C}^{-}\left(x_{i}\right)\right)+\left(v_{A}^{+}\left(x_{i}\right)-v_{C}^{+}\left(x_{i}\right)\right)\right]}{2\left(t_{1}+1\right)}\right|^{p} \\
+\left|\frac{t_{2}\left[\left(v_{A}^{-}\left(x_{i}\right)-v_{C}^{-}\left(x_{i}\right)\right)+\left(v_{A}^{+}\left(x_{i}\right)-v_{C}^{+}\left(x_{i}\right)\right)\right]-\left[\left(\mu_{A}^{-}\left(x_{i}\right)-\mu_{C}^{-}\left(x_{i}\right)\right)+\left(\mu_{A}^{+}\left(x_{i}\right)-\mu_{C}^{+}\left(x_{i}\right)\right)\right]}{2\left(t_{2}+1\right)}\right|^{p}
\end{array}\right\}^{\frac{1}{p}} .
\end{aligned}
$$


If $A \subseteq B \subseteq C$, then $\mu_{A}^{-}\left(x_{i}\right) \leq \mu_{B}^{-}\left(x_{i}\right) \leq \mu_{C}^{-}\left(x_{i}\right), \mu_{A}^{+}\left(x_{i}\right) \leq \mu_{B}^{+}\left(x_{i}\right) \leq \mu_{C}^{+}\left(x_{i}\right), v_{C}^{-}\left(x_{i}\right) \leq v_{B}^{-}\left(x_{i}\right) \leq$ $v_{A}^{-}\left(x_{i}\right)$, and $v_{C}^{+}\left(x_{i}\right) \leq v_{B}^{+}\left(x_{i}\right) \leq v_{A}^{+}\left(x_{i}\right)$. Then, we have

$$
\begin{aligned}
& \left|t_{1}\left[\left(\mu_{A}^{-}\left(x_{i}\right)-\mu_{B}^{-}\left(x_{i}\right)\right)+\left(\mu_{A}^{+}\left(x_{i}\right)-\mu_{B}^{+}\left(x_{i}\right)\right)\right]-\left[\left(v_{A}^{-}\left(x_{i}\right)-v_{B}^{-}\left(x_{i}\right)\right)+\left(v_{A}^{+}\left(x_{i}\right)-v_{B}^{+}\left(x_{i}\right)\right)\right]\right| \\
= & t_{1}\left[\left(\mu_{B}^{-}\left(x_{i}\right)-\mu_{A}^{-}\left(x_{i}\right)\right)+\left(\mu_{B}^{+}\left(x_{i}\right)-\mu_{A}^{+}\left(x_{i}\right)\right)\right]+\left[\left(v_{A}^{-}\left(x_{i}\right)-v_{B}^{-}\left(x_{i}\right)\right)+\left(v_{A}^{+}\left(x_{i}\right)-v_{B}^{+}\left(x_{i}\right)\right)\right] \\
\leq & t_{1}\left[\left(\mu_{C}^{-}\left(x_{i}\right)-\mu_{A}^{-}\left(x_{i}\right)\right)+\left(\mu_{C}^{+}\left(x_{i}\right)-\mu_{A}^{+}\left(x_{i}\right)\right)\right]+\left[\left(v_{A}^{-}\left(x_{i}\right)-v_{C}^{-}\left(x_{i}\right)\right)+\left(v_{A}^{+}\left(x_{i}\right)-v_{C}^{+}\left(x_{i}\right)\right)\right] \\
= & \left|t_{1}\left[\left(\mu_{A}^{-}\left(x_{i}\right)-\mu_{C}^{-}\left(x_{i}\right)\right)+\left(\mu_{A}^{+}\left(x_{i}\right)-\mu_{C}^{+}\left(x_{i}\right)\right)\right]-\left[\left(v_{A}^{-}\left(x_{i}\right)-v_{C}^{-}\left(x_{i}\right)\right)+\left(v_{A}^{+}\left(x_{i}\right)-v_{C}^{+}\left(x_{i}\right)\right)\right]\right| .
\end{aligned}
$$

By the same reason, we have

$$
\begin{aligned}
&\left|t_{2}\left[\left(v_{A}^{-}\left(x_{i}\right)-v_{B}^{-}\left(x_{i}\right)\right)+\left(v_{A}^{+}\left(x_{i}\right)-v_{B}^{+}\left(x_{i}\right)\right)\right]-\left[\left(\mu_{A}^{-}\left(x_{i}\right)-\mu_{B}^{-}\left(x_{i}\right)\right)+\left(\mu_{A}^{+}\left(x_{i}\right)-\mu_{B}^{+}\left(x_{i}\right)\right)\right]\right| \\
& \leq \quad\left|t_{2}\left[\left(v_{A}^{-}\left(x_{i}\right)-v_{C}^{-}\left(x_{i}\right)\right)+\left(v_{A}^{+}\left(x_{i}\right)-v_{C}^{+}\left(x_{i}\right)\right)\right]-\left[\left(\mu_{A}^{-}\left(x_{i}\right)-\mu_{C}^{-}\left(x_{i}\right)\right)+\left(\mu_{A}^{+}\left(x_{i}\right)-\mu_{C}^{+}\left(x_{i}\right)\right)\right]\right| .
\end{aligned}
$$

Therefore, $S^{p}(A, B) \geq S^{p}(A, C)$, and $S^{p}(B, C) \geq S^{p}(A, C)$.

In conclusion, $S^{p}(A, B)$ is a similarity measure between IVIFSs $A$ and $B$.

Remark 1. If interval-valued intuitionistic fuzzy sets $A$ and $B$ degenerates to intuitionistic fuzzy set, i.e., $\mu_{A}^{-}=\mu_{A}^{+}, v_{A}^{-}=v_{A}^{+}$, and $\mu_{B}^{-}=\mu_{B}^{+}, v_{B}^{-}=v_{B}^{+}$, then

$$
S^{p}(A, B)=1-\left\{\frac{1}{n} \sum_{i=1}^{n}\left|\frac{t_{1}\left(\mu_{A}-\mu_{B}\right)-\left(v_{A}-v_{B}\right)}{2\left(t_{1}+1\right)}\right|^{p}+\left|\frac{t_{2}\left(v_{A}-v_{B}\right)-\left(\mu_{A}-\mu_{B}\right)}{2\left(t_{2}+1\right)}\right|^{p}\right\}^{\frac{1}{p}}
$$

is a new similarity measure between intuitionistic fuzzy sets $A$ and $B$.

Remark 2. In the environment of IFSs, and when $t_{1}=t_{2}=t$, the proposed similarity measure

$$
S^{p}(A, B)=1-\left\{\frac{1}{2 n(t+1)^{p}} \sum_{i=1}^{n}\left(\left|t\left(\mu_{A}-\mu_{B}\right)-\left(v_{A}-v_{B}\right)\right|^{p}+\left|t\left(v_{A}-v_{B}\right)-\left(\mu_{A}-\mu_{B}\right)\right|^{p}\right)\right\}^{\frac{1}{p}}
$$

is the similarity measure between intuitionistic fuzzy sets A and B in the literature ([19]).

Example 1. Supposing that $A_{i}$ and $B_{i}$ are two IVIFSs, we can compute the similarity measures between $A_{i}$ and $B_{i}$ by different similarity measures listed in Table 1.

Table 1. Comparison of similarity measures in the environment of IVIFSs (interval-valued intuitionistic fuzzy set) (counter-intuitive cases are in bold type; $p=1$ in $S_{1}$ and $S_{2} ; p=1, t_{1}=2, t_{2}=3$ in $S^{p}$ ).

\begin{tabular}{lcccc}
\hline & $\mathbf{1}$ & $\mathbf{2}$ & $\mathbf{3}$ & $\mathbf{4}$ \\
\hline$A_{i}$ & $\langle[0.20,0.30],[0.40,0.60]\rangle$ & $\langle[0.20,0.30],[0.40,0.60]\rangle$ & $\langle[0.20,0.30],[0.30,0.50]\rangle$ & $<[0.20,0.30],[0.30,0.50]\rangle$ \\
$B_{i}$ & $\langle[0.30,0.40],[0.40,0.60]\rangle$ & $\langle[0.30,0.40],[0.30,0.50]\rangle$ & $\langle[0.30,0.40],[0.40,0.60]\rangle$ & $<[0.30,0.40],[0.30,0.50]\rangle$ \\
\hline$S_{1}[24]$ & $\mathbf{0 . 9 0}$ & $\mathbf{0 . 9 0}$ & 0.90 & 0.95 \\
$S_{2}[24]$ & $\mathbf{0 . 9 0}$ & $\mathbf{0 . 9 0}$ & $\mathbf{0 . 9 0}$ & $\mathbf{0 . 9 0}$ \\
$S_{D}[28]$ & $\mathbf{1 . 0 0}$ & 0.98 & 0.95 & 0.94 \\
$S^{p}$ & 0.95 & 0.90 & 0.80 & 0.94 \\
\hline
\end{tabular}

In Table 1, by comparing the first column and the second column, we can find that $S_{i}\left(A_{1}, B_{1}\right)=$ $S_{i}\left(A_{2}, B_{2}\right)(i=1,2)$ when $A_{1}=A_{2}, B_{1} \neq B_{2}$. Similarly, by comparing the third column and the fourth column, we can find $S_{2}\left(A_{3}, B_{3}\right)=S_{2}\left(A_{4}, B_{4}\right)$ when $A_{3}=A_{4}, B_{3} \neq B_{4}$. Therefore, we can determine that the similarity measure $S_{1}$ and $S_{2}$ is not reasonable. Meanwhile, we find that $S_{D}\left(A_{1}, B_{1}\right)=1$ when $A_{1} \neq B_{1}$, which is not satisfy the second axiom of the definition for similarity measure. Most importantly, we can observe that the proposed similarity measure $S^{p}$ can overcome these drawbacks. Therefore, our novel similarity measure for IVIFSs is more reasonable than others. 


\section{Geometric Interpretation of the Novel Similarity Measure}

In this section, we briefly interpret the proposed similarity measure and explain the functionality of parameters $t_{1}, t_{2}$ and $p$ defined in the proposed similarity measure.

Let $A=<\left[\mu_{A}^{-}, \mu_{A}^{+}\right],\left[v_{A}^{-}, v_{A}^{+}\right]>, B=<\left[\mu_{B}^{-}, \mu_{B}^{+}\right],\left[v_{B}^{-}, v_{B}^{+}\right]>$be interval-valued intuitionistic fuzzy numbers. We can split $A$ into two intuitionistic fuzzy numbers, i.e., $A^{-}=<\mu_{A}^{-}, v_{A}^{-}>$and $A^{+}=\left\langle\mu_{A}^{+}, v_{A}^{+}>\right.$. For intuitionistic fuzzy set $A^{-}, \mu_{A}^{-}$can be equal to any value in $\left[\mu_{A}^{-}, \mu_{A}^{-}+\pi_{A}^{+}\right]$and $v_{A}^{-}$can be equal to any value in $\left[v_{A}^{-}, v_{A}^{-}+\pi_{A}^{+}\right]$, where $\pi_{A}^{+}=1-\mu_{A}^{-}-v_{A}^{-}$. Similarly, $\mu_{A}^{+}$can be equal to any value in $\left[\mu_{A^{\prime}}^{+}, \mu_{A}^{+}+\pi_{A}^{-}\right]$and $v_{A}^{+}$can be equal to any value in $\left[v_{A}^{+}, v_{A}^{+}+\pi_{A}^{-}\right]$for intuitionistic fuzzy set $A^{+}$, where $\pi_{A}^{-}=1-\mu_{A}^{+}-v_{A}^{+}$. Then, the possible values for $A^{-}$and $A^{+}$illustrated in Figure 1 as the two triangles. As the center of gravity, $D^{-}$and $D^{+}$are the most informative points in the triangle $A^{-}$and $A^{+}$, respectively.

However, $<\mu_{A}^{-}+\frac{\pi_{A}^{+}}{t_{1}+1}, v_{A}^{-}+\frac{\pi_{A}^{+}}{t_{2}+1}>\left(t_{1}, t_{2} \in[1,+\infty)\right)$ can represent any point in the triangle $A^{-}$. Especially when $t_{1}=t_{2}=t,<\mu_{A}^{-}+\frac{\pi_{A}^{+}}{t+1}, v_{A}^{-}+\frac{\pi_{A}^{+}}{t+1}>$ can denote the point of middle line of the triangle bevel. In the same way, $<\mu_{A}^{+}+\frac{\pi_{A}^{-}}{t_{1}+1}, v_{A}^{+}+\frac{\pi_{A}^{-}}{t_{2}+1}>\left(t_{1}, t_{2} \in[1,+\infty)\right)$ represents any point in the triangle $A^{+}$.

The following is the calculation process:

Firstly, $A^{\prime-}=\left\langle\mu_{A}^{-}+\frac{\pi_{A}^{+}}{t_{1}+1}, v_{A}^{-}+\frac{\pi_{A}^{+}}{t_{2}+1}\right\rangle$ denotes possible points of triangle $A^{-}$. By the same token, $A^{\prime+}=\left\langle\mu_{A}^{+}+\frac{\pi_{A}^{-}}{t_{1}+1}, v_{A}^{+}+\frac{\pi_{A}^{-}}{t_{2}+1}\right\rangle$ denotes possible points of triangle $A^{+}$. Similarly, we can obtain that $B^{\prime}-\left\langle\mu_{B}^{-}+\frac{\pi_{B}^{+}}{t_{1}+1}, v_{B}^{-}+\frac{\pi_{B}^{+}}{t_{1}+1}\right\rangle$ and $B^{\prime}=\left\langle\mu_{B}^{+}+\frac{\pi_{B}^{-}}{t_{1}+1}, v_{B}^{+}+\frac{\pi_{B}^{-}}{t_{1}+1}\right\rangle$ denote any points in triangles $B^{-}$ and $B^{+}$, respectively.

Secondly, the average of $A^{\prime}-$ and $A^{\prime}+$ can be computed as follows:

$$
\left.A^{\prime \prime}=<\mu_{A}^{\prime \prime}, v_{A}^{\prime \prime}\right\rangle=\left\langle\frac{2+t_{1}\left(\mu_{A}^{-}+\mu_{A}^{+}\right)-\left(v_{A}^{-}+v_{A}^{+}\right)}{2\left(t_{1}+1\right)}, \frac{2+t_{2}\left(\mu_{A}^{-}+\mu_{A}^{+}\right)-\left(v_{A}^{-}+v_{A}^{+}\right)}{2\left(t_{2}+1\right)}\right\rangle .
$$

We can also get the mean value of $B^{\prime-}$ and $B^{\prime+}$ :

$$
B^{\prime \prime}=\left\langle\mu_{B}^{\prime \prime}, v_{B}^{\prime \prime}\right\rangle=\left\langle\frac{2+t_{1}\left(\mu_{B}^{-}+\mu_{B}^{+}\right)-\left(v_{B}^{-}+v_{B}^{+}\right)}{2\left(t_{1}+1\right)}, \frac{2+t_{2}\left(\mu_{B}^{-}+\mu_{B}^{+}\right)-\left(v_{B}^{-}+v_{B}^{+}\right)}{2\left(t_{2}+1\right)}\right\rangle .
$$

The absolute difference between $A^{\prime \prime}$ and $B^{\prime \prime}$ is calculated as follows:

$$
\begin{gathered}
\left|\mu_{A}^{\prime \prime}-\mu_{B}^{\prime \prime}\right|=\left|\frac{t_{1}\left[\left(\mu_{A}^{-}-\mu_{B}^{-}\right)+\left(\mu_{A}^{+}-\mu_{B}^{+}\right)\right]-\left[\left(v_{A}^{-}-v_{B}^{-}\right)+\left(v_{A}^{+}-v_{B}^{+}\right)\right]}{2\left(t_{1}+1\right)}\right|, \\
\left|v_{A}^{\prime \prime}-v_{B}^{\prime \prime}\right|=\left|\frac{t_{2}\left[\left(v_{A}^{-}-v_{B}^{-}\right)+\left(v_{A}^{+}-v_{B}^{+}\right)\right]-\left[\left(\mu_{A}^{-}-\mu_{B}^{-}\right)+\left(\mu_{A}^{+}-\mu_{B}^{+}\right)\right]}{2\left(t_{2}+1\right)}\right| \\
\left|\mu_{A}^{\prime \prime}-\mu_{B}^{\prime \prime}\right| \text { and }\left|v_{A}^{\prime \prime}-v_{B}^{\prime \prime}\right| \text { to the power of } p \text { is equal to the following: } \\
\left|\mu_{A}^{\prime \prime}-\mu_{B}^{\prime \prime}\right|^{p}=\frac{\left|t_{1}\left[\left(\mu_{A}^{-}-\mu_{B}^{-}\right)+\left(\mu_{A}^{+}-\mu_{B}^{+}\right)\right]-\left[\left(v_{A}^{-}-v_{B}^{-}\right)+\left(v_{A}^{+}-v_{B}^{+}\right)\right]\right|^{p}}{2^{p}\left(t_{1}+1\right)^{p}}, \\
\left|v_{A}^{\prime \prime}-v_{B}^{\prime \prime}\right|^{p}=\frac{\left|t_{2}\left[\left(v_{A}^{-}-v_{B}^{-}\right)+\left(v_{A}^{+}-v_{B}^{+}\right)\right]-\left[\left(\mu_{A}^{-}-\mu_{B}^{-}\right)+\left(\mu_{A}^{+}-\mu_{B}^{+}\right)\right]\right|^{p}}{2^{p}\left(t_{2}+1\right)^{p}}
\end{gathered}
$$


The average value of $\left|\mu_{A}^{\prime \prime}-\mu_{B}^{\prime \prime}\right|^{p}$ and $\left|v_{A}^{\prime \prime}-v_{A}^{\prime \prime}\right|^{p}$ is calculated as follows:

$$
\begin{aligned}
& \frac{1}{2}\left(\left|\mu_{A}^{\prime \prime}-\mu_{B}^{\prime \prime}\right|^{p}+\left|v_{A}^{\prime \prime}-v_{A}^{\prime \prime}\right|^{p}\right) \\
= & \frac{1}{2}\left|\frac{t_{1}\left[\left(\mu_{A}^{-}-\mu_{B}^{-}\right)+\left(\mu_{A}^{+}-\mu_{B}^{+}\right)\right]-\left[\left(v_{A}^{-}-v_{B}^{-}\right)+\left(v_{A}^{+}-v_{B}^{+}\right)\right]}{2\left(t_{1}+1\right)}\right|^{p} \\
& +\frac{1}{2}\left|\frac{t_{2}\left[\left(v_{A}^{-}-v_{B}^{-}\right)+\left(v_{A}^{+}-v_{B}^{+}\right)\right]-\left[\left(\mu_{A}^{-}-\mu_{B}^{-}\right)+\left(\mu_{A}^{+}-\mu_{B}^{+}\right)\right]}{2\left(t_{2}+1\right)}\right|^{p} .
\end{aligned}
$$

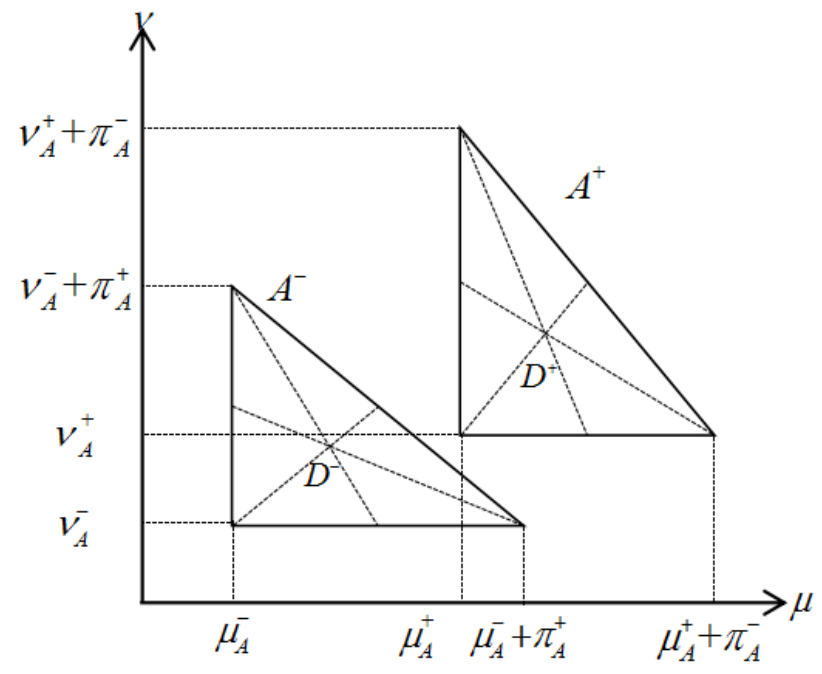

Figure 1. Possible value for $A^{-}$and $A^{+}$.

The $p$ root of the average value of $\left|\mu_{A}^{\prime \prime}-\mu_{B}^{\prime \prime}\right|^{p}$ and $\left|v_{A}^{\prime \prime}-v_{A}^{\prime \prime}\right|^{p}$ is calculated as:

$$
\left\{\frac{1}{2}\left(\left|\mu_{A}^{\prime \prime}-\mu_{B}^{\prime \prime}\right|^{p}+\left|v_{A}^{\prime \prime}-v_{B}^{\prime \prime}\right|^{p}\right)\right\}^{\frac{1}{p}}=\left\{\begin{array}{l}
\left|\frac{t_{1}\left[\left(\mu_{A}^{-}-\mu_{C}^{-}\right)+\left(\mu_{A}^{+}-\mu_{C}^{+}\right)\right]-\left[\left(v_{A}^{-}-v_{C}^{-}\right)+\left(v_{A}^{+}-v_{C}^{+}\right)\right]}{2\left(t_{1}+1\right)}\right|^{p} \\
+\left|\frac{t_{2}\left[\left(v_{A}^{-}-v_{C}^{-}\right)+\left(v_{A}^{+}-v_{C}^{+}\right)\right]-\left[\left(\mu_{A}^{-}-\mu_{C}^{-}\right)+\left(\mu_{A}^{+}-\mu_{C}^{+}\right)\right]}{2\left(t_{2}+1\right)}\right|^{p}
\end{array}\right\}^{\frac{1}{p}} .
$$

For an interval-valued intuitionistic fuzzy set instead of interval-valued intuitionistic fuzzy number, i.e., there is more than one feature in the discourse of universe, such as $X=\left\{x_{1}, x_{2}, \ldots, x_{n}\right\}$ :

$$
S^{p}(A, B)=1-\left\{\begin{array}{l}
\frac{1}{2 n} \sum_{i=1}^{n}\left|\frac{t_{1}\left[\left(\mu_{A}^{-}\left(x_{i}\right)-\mu_{B}^{-}\left(x_{i}\right)\right)+\left(\mu_{A}^{+}\left(x_{i}\right)-\mu_{B}^{+}\left(x_{i}\right)\right)\right]-\left[\left(v_{A}^{-}\left(x_{i}\right)-v_{B}^{-}\left(x_{i}\right)\right)+\left(v_{A}^{+}\left(x_{i}\right)-v_{B}^{+}\left(x_{i}\right)\right)\right]}{2\left(t_{1}+1\right)}\right|^{p} \\
+\left|\frac{t_{2}\left[\left(v_{A}^{-}\left(x_{i}\right)-v_{B}^{-}\left(x_{i}\right)\right)+\left(v_{A}^{+}\left(x_{i}\right)-v_{B}^{+}\left(x_{i}\right)\right)\right]-\left[\left(\mu_{A}^{-}\left(x_{i}\right)-\mu_{B}^{-}\left(x_{i}\right)\right)+\left(\mu_{A}^{+}\left(x_{i}\right)-\mu_{B}^{+}\left(x_{i}\right)\right)\right]}{2\left(t_{2}+1\right)}\right|^{p}
\end{array}\right\}
$$

In particular, $A^{\prime}=D^{-}=\left\langle\mu_{A}^{-}+\frac{1-\mu_{A}^{-}-v_{A}^{-}}{3}, v_{A}^{-}+\frac{1-\mu_{A}^{-}-v_{A}^{-}}{3}\right\rangle$ and $A^{\prime+}=D^{+}=$ $\left\langle\mu_{A}^{+}+\frac{1-\mu_{A}^{+}-v_{A}^{+}}{3}, v_{A}^{+}+\frac{1-\mu_{A}^{+}-v_{A}^{+}}{3}\right\rangle$ when $t_{1}=t_{2}=2$. Without a doubt, $D^{-}$and $D^{+}$are the most concentrated points of information in triangle $A^{-}$and $A^{+}$, respectively; therefore, they are also the most significant points in all possible meaningful points. 


\section{Applications}

In this section, the proposed similarity measure is used to solve the real life problems under the IVIFSs environment and obtained results have been compared with some existing similarity measures.

\subsection{Pattern Recognition}

\subsubsection{Algorithms for Pattern Recognition}

Letting $X=\left\{x_{1}, x_{2}, \ldots, x_{n}\right\}$ be a finite universe of discourse, there exists $m$ patterns which are denoted by IVIFSs $A_{j}=\left\{<x_{1},\left[\mu_{A_{j}}^{-}\left(x_{1}\right), \mu_{A_{j}}^{+}\left(x_{1}\right)\right],\left[v_{A_{j}}^{-}\left(x_{1}\right), v_{A_{j}}^{+}\left(x_{1}\right)\right]>, \ldots,<\right.$ $\left.x_{1},\left[\mu_{A_{j}}^{-}\left(x_{n}\right), \mu_{A_{j}}^{+}\left(x_{n}\right)\right],\left[v_{A_{j}}^{-}\left(x_{n}\right), v_{A_{j}}^{+}\left(x_{n}\right)\right]>\mid x_{1}, \ldots, x_{n} \in X\right\}(j=1,2, \ldots, m)$ and there is a test sample to be classified which is denoted by an IVIFS $B=\left\{<x_{1},\left[\mu_{B}^{-}\left(x_{1}\right), \mu_{B}^{+}\left(x_{1}\right)\right],\left[v_{B}^{-}\left(x_{1}\right), v_{B}^{+}\left(x_{1}\right)\right]>, \ldots,<\right.$ $\left.x_{1},\left[\mu_{B}^{-}\left(x_{n}\right), \mu_{B}^{+}\left(x_{n}\right)\right],\left[v_{B}^{-}\left(x_{n}\right), v_{B}^{+}\left(x_{n}\right)\right]>\mid x_{1}, \ldots, x_{n} \in X\right\}$. The recognition process is as follows:

Step 1. Calculate the similarity measure $S\left(B, A_{j}\right)$ between $B$ and $A_{j}(j=1, \ldots, m)$.

Step 2. Choose the maximum one $S\left(B, A_{j_{0}}\right)$ from $S\left(B, A_{j}\right)(j=1,2, \ldots, m)$, i.e., $S\left(B, A_{j_{0}}\right)=$ $\max _{1 \leq j \leq m} S\left(B, A_{j}\right)$. Then, the test sample $B$ is classified the pattern $A_{j_{0}}$.

\subsubsection{Applications for Pattern Recognition}

Example 2. Assume that there are four classes of ores $A_{i}(i=1,2,3,4)$ in the area developed by a coal mine company, for which the related feature information are expressed by IVIFSs, and $A_{i}=$ $\left\{<x_{1},\left[\mu_{A_{i}}^{-}\left(x_{1}\right), \mu_{A_{i}}^{+}\left(x_{1}\right)\right],\left[v_{A_{i}}^{-}\left(x_{1}\right), v_{A_{i}}^{+}\left(x_{1}\right)\right]>, \ldots,<x_{4},\left[\mu_{A_{i}}^{-}\left(x_{4}\right), \mu_{A_{i}}^{+}\left(x_{4}\right)\right],\left[v_{A_{i}}^{-}\left(x_{4}\right), v_{A_{i}}^{+}\left(x_{4}\right)\right]>\right.$ $\left.\mid x_{1}, x_{2}, x_{3}, x_{4} \in X\right\}$, which are presented in Table 2. Now, there is an unknown ore $B$ and our aim is to classify $B$ into the four kinds of ores above.

Table 2. Feature matrix of $A_{1}, A_{2}, A_{3}, A_{4}$ and $B$.

\begin{tabular}{ccccc}
\hline & Feature1 & Feature2 & Feature3 & Feature4 \\
\hline$A_{1}$ & $<[0.10,0.50],[0.20,0.30]>$ & $<[0.10,0.30],[0.00,0.20]>$ & $<[0.30,0.50],[0.20,0.40]>$ & $<[0.20,0.50],[0.10,0.30]>$ \\
$A_{2}$ & $<[0.20,0.40],[0.15,0.35]>$ & $<[0.20,0.20],[0.05,0.15]>$ & $<[0.20,0.60],[0.30,0.30]>$ & $<[0.30,0.40],[0.15,0.25]>$ \\
$A_{3}$ & $<[0.15,0.30],[0.30,0.40]>$ & $<[0.20,0.40],[0.50,0.60]>$ & $<[0.50,0.60],[0.15,0.35]>$ & $<[0.25,0.45],[0.30,0.40]>$ \\
$A_{4}$ & $<[0.20,0.35],[0.10,0.65]>$ & $<[0.35,0.60],[0.05,0.30]>$ & $<[0.15,0.30],[0.40,0.55]>$ & $<[0.15,0.25],[0.45,0.55]>$ \\
$B$ & $<[0.30,0.40],[0.10,0.50]>$ & $<[0.10,0.40],[0.25,0.40]>$ & $<[0.20,0.30],[0.10,0.35]>$ & $<[0.15,0.40],[0.20,0.50]>$ \\
\hline
\end{tabular}

Compute the similarity measures $S\left(A_{i}, B\right)$ between $B$ and $A_{i}$. By analyzing the computed results in Table 3 , we can easily see that, if $S_{1}$ is used for pattern recognition, we can obtain that $S_{1}\left(A_{1}, B\right)=S_{1}\left(A_{2}, B\right)=$ $S_{1}\left(A_{4}, B\right)>S_{1}\left(A_{3}, B\right)$. In this way, we can not classify the sample $B$ into a certain pattern accurately. If $S_{W}$ is used for pattern recognition, we can obtain that $S_{W}\left(A_{2}, B\right)=S_{W}\left(A_{4}, B\right)>S_{W}\left(A_{1}, B\right)=S_{W}\left(A_{3}, B\right)$. In this way, we can not make sure if the sample $B$ belongs to one of $A_{2}$ and $A_{4}$. If we use $S_{D}$ for pattern recognition, we can get $S\left(A_{3}, B\right)=S\left(A_{4}, B\right)>S\left(A_{2}, B\right)>S\left(A_{1}, B\right)$. In this way, we can not classify the sample $B$ into one of $A_{3}$ and $A_{4}$. If we use $S^{p}$ for pattern recognition, we can get $S\left(A_{1}, B\right)>S\left(A_{2}, B\right)>S\left(A_{3}, B\right)>S\left(A_{4}, B\right)$. According to the principle of recognition, $S_{2}$ and $S^{p}$ can get the same recognition result, i.e., the sample $B$ can be classified into the pattern $A_{3}$. However, we can not distinguish which one is bigger between $A_{2}$ and $A_{4}$ when using $S_{2}$ to calculate the similarity measure. Therefore, we can assign the sample $B$ to the pattern $A_{3}$. 
Table 3. Pattern recognition result under different similarity measures (counter-intuitive cases are in bold type; $p=1$ in $S_{1}$ and $S_{2} ; p=1, t_{1}=2, t_{2}=3$ in $S^{p} ;$ N.A. means method is not applicable).

\begin{tabular}{lccccc}
\hline & $S\left(A_{\mathbf{1}}, \boldsymbol{B}\right)$ & $S\left(\boldsymbol{A}_{\mathbf{2}}, \boldsymbol{B}\right)$ & $S\left(\boldsymbol{A}_{\mathbf{3}}, \boldsymbol{B}\right)$ & $S\left(\boldsymbol{A}_{\mathbf{4}}, \boldsymbol{B}\right)$ & Classification Results \\
\hline$S_{1}[24]$ & $\mathbf{0 . 8 7}$ & $\mathbf{0 . 8 7}$ & 0.86 & $\mathbf{0 . 8 7}$ & N.A. \\
$S_{2}[24]$ & 0.75 & $\mathbf{0 . 7 6}$ & 0.79 & $\mathbf{0 . 7 6}$ & $A_{3}$ \\
$S_{W}[25]$ & 0.78 & $\mathbf{0 . 7 9}$ & 0.78 & $\mathbf{0 . 7 9}$ & N.A. \\
$S_{D}[28]$ & 0.82 & 0.86 & $\mathbf{0 . 8 8}$ & $\mathbf{0 . 8 8}$ & N.A. \\
$S^{p}$ & 0.82 & 0.81 & 0.88 & 0.75 & $A_{3}$ \\
\hline
\end{tabular}

Example 3 ([30]). In this example, a pattern recognition example about classification of building materials is used to illustrate the proposed similarity measure. Suppose that there are four classes of building material, which are denoted by the IVIFSs $A_{j}=\left\{<x_{1},\left[\mu_{A_{j}}^{-}\left(x_{1}\right), \mu_{A_{j}}^{+}\left(x_{1}\right)\right],\left[v_{A_{j}}^{-}\left(x_{1}\right), v_{A_{j}}^{+}\left(x_{1}\right)\right]>, \ldots,<\right.$ $\left.x_{12},\left[\mu_{A_{j}}^{-}\left(x_{12}\right), \mu_{A_{j}}^{+}\left(x_{12}\right)\right],\left[v_{A_{j}}^{-}\left(x_{12}\right), v_{A_{j}}^{+}\left(x_{12}\right)\right]>\mid x_{1}, \ldots, x_{12} \in X\right\}(j=1, \ldots, 4)$ in the feature space $X=\left\{x_{1}, x_{2}, \ldots, x_{12}\right\}$, and there is an unknown pattern $B$ :

$$
\begin{aligned}
& \left.A_{1}=\left\{<x_{1},[0.1,0.2],[0.5,0.6]\right\rangle,<x_{2},[0.1,0.2],[0.7,0.8]\right\rangle,\left\langle x_{3},[0.5,0.6],[0.3,0.4]\right\rangle \text {, } \\
& \left\langle x_{4},[0.8,0.9],[0.0,0.1]\right\rangle,\left\langle x_{5},[0.4,0.5],[0.3,0.4]\right\rangle,\left\langle x_{6},[0.0,0.1],[0.8,0.9]\right\rangle \text {, } \\
& \left\langle x_{7},[0.3,0.4],[0.5,0.6]\right\rangle,\left\langle x_{8},[1.0,1.0],[0.0,0.0]\right\rangle,\left\langle x_{9},[0.2,0.3],[0.6,0.7]\right\rangle \text {, } \\
& \left.\left.<x_{10},[0.4,0.5],[0.4,0.5]>,<x_{11},[0.7,0.8],[0.1,0.2]\right\rangle,<x_{12},[0.4,0.5],[0.4,0.5]>\right\} \text {, } \\
& A_{2}=\left\{\left\langle x_{1},[0.5,0.6],[0.3,0.4]\right\rangle,\left\langle x_{2},[0.6,0.7],[0.1,0.2]\right\rangle,\left\langle x_{3},[1.0,1.0],[0.0,0.0]\right\rangle,\right. \\
& \left\langle x_{4},[0.1,0.2],[0.6,0.7]\right\rangle,\left\langle x_{5},[0.0,0.1],[0.8,0.9]\right\rangle,\left\langle x_{6},[0.7,0.8],[0.1,0.2]\right\rangle \text {, } \\
& \left\langle x_{7},[0.5,0.6],[0.3,0.4]\right\rangle,\left\langle x_{8},[0.6,0.7],[0.2,0.3]\right\rangle,\left\langle x_{9},[1.0,1.0],[0.0,0.0]\right\rangle \text {, } \\
& \left.<x_{10},[0.1,0.2],[0.7,0.8]>,<x_{11},[0.0,0.1],[0.8,0.9]>,<x_{12},[0.7,0.8],[0.1,0.2]>\right\} \text {, } \\
& A_{3}=\left\{\left\langle x_{1},[0.4,0.5],[0.3,0.4]\right\rangle,\left\langle x_{2},[0.6,0.7],[0.2,0.3]\right\rangle,\left\langle x_{3},[0.9,1.0],[0.0,0.0]\right\rangle,\right. \\
& \left\langle x_{4},[0.0,0.1],[0.8,0.9]\right\rangle,\left\langle x_{5},[0.0,0.1],[0.8,0.9]\right\rangle,\left\langle x_{6},[0.6,0.7],[0.2,0.3]\right\rangle \text {, } \\
& <x_{7},[0.1,0.2],[0.7,0.8]>,<x_{8},[0.2,0.3],[0.6,0.7]>,<x_{9},[0.5,0.6],[0.2,0.4]>\text {, } \\
& \left.\left\langle x_{10},[1.0,1.0],[0.0,0.0]\right\rangle,\left\langle x_{11},[0.3,0.4],[0.4,0.5]\right\rangle,\left\langle x_{12},[0.0,0.1],[0.8,0.9]\right\rangle\right\} \text {, } \\
& A_{4}=\left\{\left\langle x_{1},[1.0,1.0],[0.0,0.0]\right\rangle,\left\langle x_{2},[1.0,1.0],[0.0,0.0]\right\rangle,\left\langle x_{3},[0.8,0.9],[0.0,0.1]\right\rangle,\right. \\
& \left\langle x_{4},[0.7,0.8],[0.1,0.2]\right\rangle,\left\langle x_{5},[0.0,0.1],[0.7,0.9]\right\rangle,\left\langle x_{6},[0.0,0.1],[0.8,0.9]\right\rangle \text {, } \\
& <x_{7},[0.1,0.2],[0.7,0.8]>,<x_{8},[0.1,0.2],[0.7,0.8]>,<x_{9},[0.4,0.5],[0.3,0.4]>\text {, } \\
& \left.\left.<x_{10},[1.0,1.0],[0.0,0.0]\right\rangle,\left\langle x_{11},[0.3,0.4],[0.4,0.5]\right\rangle,\left\langle x_{12},[0.0,0.1],[0.8,0.9]\right\rangle\right\} \text {, } \\
& \left.B=\left\{<x_{1},[0.9,1.0],[0.0,0.0]\right\rangle,<x_{2},[0.9,1.0],[0.0,0.0]\right\rangle,\left\langle x_{3},[0.7,0.8],[0.1,0.2]\right\rangle, \\
& \left.<x_{4},[0.6,0.7],[0.1,0.2]\right\rangle,\left\langle x_{5},[0.0,0.1],[0.8,0.9]\right\rangle,\left\langle x_{6},[0.1,0.2],[0.7,0.8]\right\rangle \text {, } \\
& <x_{7},[0.1,0.2],[0.7,0.8]>,<x_{8},[0.1,0.2],[0.7,0.8]>,\left\langle x_{9},[0.4,0.5],[0.3,0.4]>\right.\text {, } \\
& \left.\left.\left.<x_{10},[1.0,1.0],[0.0,0.0]\right\rangle,<x_{11},[0.3,0.4],[0.4,0.5]\right\rangle,<x_{12},[0.0,0.1],[0.7,0.9]>\right\} \text {. }
\end{aligned}
$$

Calculate the similarity measure $S\left(A_{j}, B\right)$ between IVIFSs $A_{j}(j=1,2,3,4)$ and B by use of Formulas (1)-(5). It is obvious that the similarity measure in the literature ([30]) is the special case of $S_{1}$ and $S_{2}$, and the computed result is the same as ([30]). According to Table 4 and the recognition principle, the unknown pattern can be classified properly in $A_{4}$ by the computation of similarity measure. This conclusion coincides with that in [30]. 
Table 4. Pattern recognition results under different similarity measures (counter-intuitive cases are in bold type; $p=1$ in $S_{1}$ and $S_{2}, p=1, t_{1}=2, t_{2}=3$ in $S^{p}$ ).

\begin{tabular}{lccccc}
\hline & $S\left(\boldsymbol{A}_{\mathbf{1}}, \boldsymbol{B}\right)$ & $\boldsymbol{S}\left(\boldsymbol{A}_{\mathbf{2}} \boldsymbol{B}\right)$ & $\boldsymbol{S}\left(\boldsymbol{A}_{\mathbf{3}}, \boldsymbol{B}\right)$ & $\boldsymbol{S}\left(\boldsymbol{A}_{\mathbf{4}}, \boldsymbol{B}\right)$ & Recognition Results \\
\hline$S_{1}[24]$ & 0.59 & 0.58 & 0.81 & 0.97 & $A_{4}$ \\
$S_{2}[24]$ & $\mathbf{0 . 5 3}$ & $\mathbf{0 . 5 3}$ & 0.79 & 0.94 & $A_{4}$ \\
$S_{W}[25]$ & 0.48 & 0.47 & 0.74 & 0.94 & $A_{4}$ \\
$S_{D}[28]$ & 0.64 & 0.56 & 0.83 & 0.98 & $A_{4}$ \\
$S^{p}$ & 0.60 & 0.58 & 0.85 & 0.97 & $A_{4}$ \\
\hline
\end{tabular}

\subsection{Applications for Medical Diagnosis}

Researchers proposed a lot of methods from different points of view to deal with problems of medical diagnosis. Refs. [27,31-33] presented several ways to deal with the problems of medical diagnosis. In this section, the methods of pattern recognition are used for solving medical diagnosis problems, i.e., patients are unknown test samples, diseases are several patterns, and the symptom set is the set universe of discourse. Our aim is to classify patients in one of the illnesses, respectively.

Example 4. Let $A=\left\{A_{1}\right.$ (Viral fever), $A_{2}$ (Typhoid), $A_{3}$ (Pneumonia), $A_{4}$ (Stomach problem) $\}$ be a set of diagnoses and $X=\left\{x_{1}\right.$ (Temperature), $x_{2}$ (Cough), $x_{3}$ (Headache), $x_{4}$ (Stomach pain) $\}$ be a set of symptoms. The disease-symptom matrix that is represented by IVIFSs is listed in Table 5.

Table 5. Disease-symptom matrix.

\begin{tabular}{lllll}
\hline & $x_{1}$ (Temperature) & $x_{2}$ (Cough) & $x_{3}$ (Headache) & $x_{4}$ (Stomach Pain) \\
\hline$A_{1}$ (Viral fever) & $<[0.8,0.9],[0.0,0.1]>$ & $<[0.7,0.8],[0.1,0.2]>$ & $<[0.5,0.6],[0.2,0.3]>$ & $<[0.6,0.8],[0.1,0.2]>$ \\
$A_{2}$ (Typhoid) & $<[0.5,0.6],[0.1,0.3]>$ & $<[0.8,0.9],[0.0,0.1]>$ & $<[0.6,0.8,,[0.1,0.2]>$ & $<[0.4,0.6],[0.1,0.2]>$ \\
$A_{3}$ (Pneumonia) & $<[0.7,0.8],[0.1,0.2]>$ & $<[0.7,0.9],[0.0,0.1]>$ & $<[0.4,0.6],[0.2,0.4]>$ & $<[0.3,0.5],[0.2,0.4]>$ \\
$A_{4}$ (Stomach problem) & $<[0.8,0.9],[0.0,0.1]>$ & $<[0.7,0.8],[0.1,0.2]>$ & $<[0.7,0.9],[0.0,0.1]>$ & $<[0.8,0.9],[0.0,0.1]>$ \\
\hline
\end{tabular}

Suppose the patient $B$ can be represented as:

$B=\left\{<x_{1},[0.4,0.5],[0.1,0.2]>,<x_{2},[0.7,0.8],[0.1,0.2]>,<x_{3},[0.9,0.9],[0.0,0.1]>,<\right.$ $\left.x_{4},[0.3,0.5],[0.2,0.4]>\right\}$.

Our aim is to classify the patient $B$ in one of the illnesses $A_{1}, A_{2}, A_{3}$ and $A_{4}$. Then, we can have the following results in the environment of IVIFSs, which are listed in Table 6.

Table 6. Computed results under different similarity measures (counter-intuitive cases are in bold type; $p=1$ in $S_{1}$ and $S_{2} ; p=1, t_{1}=2, t_{2}=3$ in $S^{p}$ ).

\begin{tabular}{lccccc}
\hline & $S\left(\boldsymbol{A}_{\mathbf{1}} \boldsymbol{B}\right)$ & $\boldsymbol{S}\left(\boldsymbol{A}_{\mathbf{2}} \boldsymbol{B}\right)$ & $\boldsymbol{S}\left(\boldsymbol{A}_{\mathbf{3}}, \boldsymbol{B}\right)$ & $\boldsymbol{S}\left(\boldsymbol{A}_{\mathbf{4}}, \boldsymbol{B}\right)$ & Recognition Result \\
\hline$S_{1}[24]$ & 0.81 & 0.89 & 0.86 & 0.84 & $A_{2}$ \\
$S_{2}[24]$ & $\mathbf{0 . 7 3}$ & 0.80 & 0.78 & $\mathbf{0 . 7 3}$ & $A_{2}$ \\
$S_{W}[25]$ & 0.82 & 0.80 & 0.79 & 0.77 & $A_{2}$ \\
$S_{D}[28]$ & 0.82 & 0.91 & 0.86 & 0.84 & $A_{2}$ \\
$S^{p}$ & 0.83 & 0.89 & 0.87 & 0.85 & $A_{2}$ \\
\hline
\end{tabular}

Considering the recognition principle of the maximum similarity degree for the IVIFSs, we can obtain the consequence that the similarity measure between $A_{2}$ and $B$ is the largest one. However, the similarity measures $S_{2}$ could not distinguish which one is bigger between $A_{1}$ and $A_{4}$. Thus, we can classify the patient $B$ to illness $A_{2}$ due to the recognition principle. Therefore, we can diagnose that the patient's disease is typhoid.

\section{Conclusions}

In this paper, a novel similarity measure for IVIFSs is proposed, which is obtained by splitting an IVIFS into two IFSs and computing the average value of the $p$ power of any points in two triangles composed of the two intuitionistic fuzzy sets. Its superiority is presented by comparing the developed 
similarity measure with some existing similarity measures. Thus, we can use the similarity measure to deal with the problems with vagueness and uncertainty. For example, pattern recognition, medical diagnosis, game theory and so on.

In fact, we can choose different values of the three parameters $\left(t_{1}, t_{2}\right.$ and $p$ in Formula (5)) when facing different problems. However, there are some difficulties when choosing the value of parameters. This is also a problem to be solved in the future.

Author Contributions: M.L. initiated the research and provide the framework of this paper. J.L. wrote and complete this paper with M.L.'s validity and helpful suggeations.

Acknowledgments: The authors would like to thank the reviewers for their valuable comments and suggestions. This research was supported by the National Natural Science Foundation of China (Grant No. 61773019).

Conflicts of Interest: The authors declare no conflict of interest.

\section{References}

1. Zadeh, L.A. Fuzzy sets. Inf. Control 1965, 8, 338-353. [CrossRef]

2. Atanassov, K.T.; Rangasamy, P. Intuitionistic Fuzzy Sets. Fuzzy Sets Syst. 1986, 20, 87-96. [CrossRef]

3. Gau, W.L.; Buehrer, D.J. Vague sets. IEEE Trans. Syst. Man Cybern. 1993, 23, 610-614. [CrossRef]

4. Bustince, H.; Burillo, P. Vague sets are intuitionistic fuzzy sets. Fuzzy Sets Syst. 1996, 79, 403-405. [CrossRef]

5. Smarandache, F. A Unifiying Field in Logics: Neutrosophic Logic, Neutrosophy, Neutrosophic Set, Neutrosophic Probability; American Research Press: Rehoboth, DE, USA, 1999.

6. Zhang, X.H.; Smarandache, F.; Liang, X.L. Neutrosophic duplet semi-group and cancellable neutrosophic triplet groups. Symmetry 2017, 9, 275. [CrossRef]

7. Zhang, X.H.; Bo, C.X.; Smarandache, F.; Park, C. New operations of totally dependent- neutrosophic sets and totally dependent-neutrosophic soft sets. Symmetry 2018, 10, 187. [CrossRef]

8. Chen, S.M. Similarity measures between vague sets and between elements. IEEE Trans. Syst. Man Cybern. Part B Cybern. 1997, 27, 153-158. [CrossRef] [PubMed]

9. Hong, D.H.; Kim, C. A note on similarity measures between vague sets and between elements. Inf. Sci. 1999, 115, 83-96. [CrossRef]

10. Szmidt, E.; Kacprzyk, J. Distances between intuitionistic fuzzy sets. Fuzzy Sets Syst. 2000, 114, 505-518. [CrossRef]

11. Wang, W.Q.; Xin, X.L. Distance measure between intuitionistic fuzzy sets. Pattern Recognit. Lett. 2005, 26, 2063-2069. [CrossRef]

12. Grzegorzewski, P. Distances between intuitionistic fuzzy sets and/or interval-valued fuzzy sets based on the Hausdorff metric. Fuzzy Sets Syst. 2004, 148, 319-328. [CrossRef]

13. Chen, T.Y. A note on distances between intuitionistic fuzzy sets and/or interval-valued fuzzy sets based on the Hausdorff metric. Fuzzy Sets Syst. 2007, 158, 2523-2525. [CrossRef]

14. Li, D.F.; Cheng, C. New similarity measures of intuitionistic fuzzy sets and application to pattern recognitions. Pattern Recognit. Lett. 2002, 23, 221-225. [CrossRef]

15. Mitchell, H.B. On the Dengfeng-Chuntian similarity measure and its application to pattern recognition. Pattern Recognit. Lett. 2003, 24, 3101-3104. [CrossRef]

16. Liang, Z.Z.; Shi, P. Similarity measures on intuitionistic fuzzy sets. Pattern Recognit. Lett. 2003, 24, 2687-2693. [CrossRef]

17. Ye, J. Cosine similarity measures for intuitionistic fuzzy sets and their applications. Math. Comput. Model. 2011, 53, 91-97. [CrossRef]

18. $\mathrm{Xu}, \mathrm{Z}$.S. Some similarity measures of intuitionistic fuzzy sets and their applications to multiple attribute decision making. Fuzzy Optim. Decis. Mak. 2007, 6, 109-121. [CrossRef]

19. Boran, F.E.; Akay, D. A biparametric similarity measure on intuitionistic fuzzy sets with applications to pattern recognition. Inf. Sci. 2014, 255, 45-57. [CrossRef]

20. Zhang, H.M.; Yu, L.Y. New distance measures between intuitionistic fuzzy sets and interval-valued fuzzy sets. Inf. Sci. 2013, 245, 181-196. [CrossRef]

21. Luo, M.X.; Zhao, R.R. A distance measure between intuitionistic fuzzy sets and its application in medical diagnosis. Artif. Intell. Med. 2018, 89, 34-39. [CrossRef] [PubMed] 
22. Atanassov, K.T.; Gargov, G. Interval-valued intuitionistic fuzzy sets. Fuzzy Sets Syst. 1989, 31, $343-349$. [CrossRef]

23. Liu, X.D.; Zheng, S.H.; Xiong, F.L. Entropy and subsethood for general interval-valued intuitionistic fuzzy sets. Int. Conf. Fuzzy Syst. Knowl. Discov. 2005, 42-52. [CrossRef]

24. Xu, Z.S.; Chen, J. An overview of distance and simiarity measures of intuitionistic fuzzy sets. Int. J. Uncertain. Fuzziness Knowl.-Based Syst. 2008, 16, 529-555. [CrossRef]

25. Wei, C.P.; Wang, P.; Zhang, Y.Z. Entropy, similarity measure of interval-valued intuitionistic fuzzy sets and their applications. Inf. Sci. 2011, 181, 4273-4286. [CrossRef]

26. Singh, P. A New Method on Measure of Similarity between Interval-Valued Intuitionistic Fuzzy Sets for Pattern Recognition. J. Appl. Comput. Math. 2012, 1. [CrossRef]

27. Khalaf, M. Medical diagnosis via interval valued intuitionistic fuzzy sets. Ann. Fuzzy Math. Inf. 2013, $6,245-249$.

28. Dhivya, J.; Sridevi, B. Similarity measure between interval-valued intuitionistic fzzy sets and their applications to medical diagnosis and pattern recognition. Int. J. Math. Arch. 2018, 9, 58-65.

29. Meng, F.Y.; Chen, X. Entropy and similarity measure for Atannasov's interval-valued intuitionistic fuzzy sets and their application. Fuzzy Optim. Decis. Mak. 2015, 15, 75-101. [CrossRef]

30. Xu, Z.S. On similarity measures of interval-valued intuitionistic fuzzy sets and their application to pattern recognitions. J. Southeast Univ. 2007, 23, 027.

31. Mathew, T.J.; Sherly, E.; Alcantud, J.C.R. A multimodal adaptive approach on soft set based diagnostic risk prediction system. J. Intell. Fuzzy Syst. 2018, 34, 1609-1618. [CrossRef]

32. Celik, Y.; Yamak, S. Fuzzy soft set theory applied to medical diagnosis using fuzzy arithmetic operations. J. Inequal. Appl. 2013, 1, 82. [CrossRef]

33. De, S.K.; Biswas, R.; Roy, A.R. An application of intuitionistic fuzzy sets in medical diagnosis. Fuzzy Sets Syst. 2001, 117, 209-213. [CrossRef]

(C) 2018 by the authors. Licensee MDPI, Basel, Switzerland. This article is an open access article distributed under the terms and conditions of the Creative Commons Attribution (CC BY) license (http:/ / creativecommons.org/licenses/by/4.0/). 